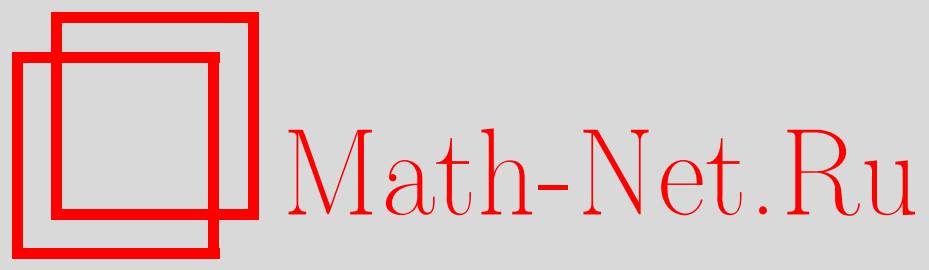

Ю. И. Любич, Ультранормальный случай проблемы Бернштейна, Функи. анализ и его прил., 1997, том 31, выпуск 1, 77-80

DOI: https://doi.org/10.4213/faa449

Использование Общероссийского математического портала Math$\mathrm{Net.Ru}$ подразумевает, что вы прочитали и согласны с пользовательским соглашением

http://www . mathnet.ru/rus/agreement

Параметры загрузки:

IP: 3.81 .55 .215

26 апреля 2023 г., 13:08:38

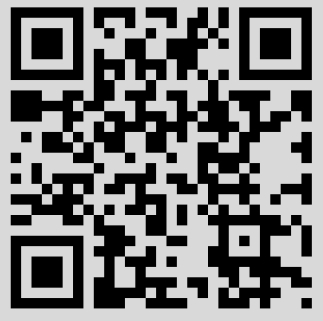


ющую деформацию $\pi\left(L_{\lambda}\right)$ кривой $\{f=0\}=\pi(L)$. Эта деформация может быть реализована вещественной деформацией $\tilde{f}=f_{\lambda}$ ростка $f=f_{0}$. У ростка функции $f_{\lambda}$ в начале координат число Милнора на два меньше, чем у ростка $f$. При любом $\lambda$ росток кривой $\left\{f_{\lambda}=0\right\}$ в начале координат неприводим (состоит из одной вещественной компоненты при $\lambda \in \mathbb{R}$ ). Можно показать, что при $\lambda>0$ функция $f_{\lambda}$ не имеет вещественных критических точек, отличных от начала координат. Это завершает рассмотрение случая (ii).

Случай (iii). В окрестности точки пересечения $L$ с исключительным дивизором $D$ существуют такие координаты $(x, y)$, в которых $D$ совпадает с объединением координатных осей, а $L$ задается уравнением $(y-x)^{2}-x^{3}=0$ (это вытекает, например, из вида нормальной формы особенности $X_{1,1}$; см. [1, том I]). В этих координатах функция $f \circ \pi$, индуцированная функцией $f$ на пространстве модификации $X$, имеет вид $u(x, y) x^{m} y^{n}\left((y-x)^{2}-x^{3}\right)$, где $u(0,0) \neq 0$. Мы предполагаем, что $m \geqslant n>0$. Пусть $L_{\lambda}=\left\{(y-x-\lambda / 2)^{2}-x^{3}-\lambda x=0\right\}$ - деформация кривой $L=\left\{(y-x)^{2}-x^{3}=0\right\}$. При $\lambda \neq 0$ кривая $L_{\lambda}$ является кривой рода 1 с одной компонентой края. Пусть $f_{\lambda}$ - вещественная деформация ростка $f$, соответствующая деформации $\pi\left(L_{\lambda}\right)$ кривой $\{f=0\}=\pi(L)$. У ростка функции $f_{\lambda}$ в начале координат число Милнора на четыре меньше, чем у ростка $f$. При любом вещественном $\lambda \neq 0$ росток кривой $\left\{f_{\lambda}=0\right\}$ в начале координат имеет одну вещественную компоненту и одну пару комплексносопряженных компонент (соответствующих пересечениям кривой $L_{\lambda}$ с компонентами $\{x=0\}$ и $\{y=0\}$ исключительного дивизора $D$ соответственно). Можно показать, что при $\lambda>0$ функция $f_{\lambda}$ не имеет вещественных критических точек, отличных от начала координат. Это завершает рассмотрение случая (iii) и доказательство теоремы.

Автор благодарен Ю. В. Чеканову и И. Луенго (I. Luengo) за полезные обсуждения.

\title{
ЛИТЕРАТУРА
}

1. Арнольд В. И., Варченко А. Н., Гусейн-Заде С. М. Особенности дифференцируемых отображений. Наука, М., т. I, 1982; т. II, 1984. 2. Милнор Дж. Особые точки комплексных гиперповерхностей. Мир, М., 1971.

Московский государственный университет и Независимый московский университет

Поступило в редакцию 28 февраля 1996 г.

\section{Ультранормальный случай проблемы Бернштейна}

\author{
(c) 1997. Ю. И. Люьич
}

Проблема Бернштейна касается одного из фундаментальных положений популяционной генетики, которое называется принципом стачионарности. Этот принцип реализуется в условиях закона Менделя, однако он согласуется и со многими другими законами наследственности. Принцип стационарности был введен С. Н. Бернштейном [1] в 1922 г. в качестве аксиоматической основы описа- 
ния всех допустимых модификаций закона Менделя. Адекватная математическая проблема звучит так.

Пусть $\Delta^{n-1}$ - координатный симплекс в $\mathbb{R}^{n}$, т. е.

$$
\Delta^{n-1}=\left\{x \in \mathbb{R}^{n}: s(x) \equiv \sum x_{i}=1, x=\left(x_{1}, \ldots, x_{n}\right) \geqslant 0\right\}
$$

Рассмотрим квадратичное отображение $V: \Delta^{n-1} \rightarrow \Delta^{n-1}$,

$$
x_{j}^{\prime} \equiv(V x)_{j}=\sum_{i, k=1}^{n} p_{i k, j} x_{i} x_{k} \quad(1 \leqslant j \leqslant n),
$$

cmохастическое в том смысле, что $p_{i k, j} \geqslant 0$ и $\sum_{j} p_{i k, j}=1$. С биологической точки зрения $V$ - это эволюиионный оператор некоторой бесконечной панмиксной селективно нейтральной популяции, а $p_{i k, j}$ - это коэффициенты наследственности, причем $p_{k i, j}=p_{i k, j}$, и это означает, что наследственность не зависит от дифференциации полов, см. [6, 8].

Отображение $V$ называется бернштейновским, если $V^{2}=V$. Это свойство как раз и составляет принцип стационарности, а общая проблема Бернштейна заключается в явном описании всех бернштейновских отображений.

Случай $n \leqslant 2$ тривиален с математической точки зрения и не представляет интереса с биологической. Бернштейн [2-4] решил сформулированную проблему в случае $n=3$ и получил отдельные результаты для $n \geqslant 4$. В частности, классическое отображение Харди-Вайнберга $x_{1}^{\prime}=p^{2}, x_{2}^{\prime}=q^{2}, x_{3}^{\prime}=2 p q$ с $p=x_{1}+\frac{1}{2} x_{3}$ и $q=x_{2}+\frac{1}{2} x_{3}$ содержится в списке Бернштейна, но без промежуточных переменных $p$ и $q$. Фактически это отображение - прямое следствие закона Менделя $[5,9]$. С биологической точки зрения линейные формы $p, q$ суть вероятности аллельных генов, скажем $\mathbf{A}$ и $\mathbf{a}$, если генотипы $\mathbf{A} \mathbf{A}$, $\mathbf{a}, \mathbf{A a}$ индивидуумов в популяции имеют вероятности соответственно $x_{1}, x_{2}, x_{3}$. Указанные формы инвариантны в том смысле, что $p(V x)=p(x), q(V x)=q(x)$, и это означает, что при переходе от родителей к потомкам гены не исчезают и не возникают. В силу этих законов сохранения указанное отображение автоматически является бернштейновским.

В наших работах 1971-77 гг. (первая из них - статья [6]; систематическое изложение и полные ссылки даны в книге [8]) проблема Бернштейна была решена для всех $n$ в дополнительном предположении регулярности. Регулярность означает, что $V x$ зависит только от значений $f(x)$, где $f$ пробегает совокупность всех инвариантных линейных форм. (Без этого свойства, по-видимому, невозможна интерпретация в терминах генов.) В упомянутых исследованиях очень важную роль играет алгебра $A_{V}$ со структурными константами $p_{i k, j}$. Так как $V x=x^{2}$, то бернштейновское свойство отображения $V$ равносильно тождеству

$$
\left(x^{2}\right)^{2}=s^{2}(x) x^{2} .
$$

Тождество (1) по определению означает, что $A_{V}$ есть алгебра Бернщтейна относительно алгебраического гомоморфизма $s: A_{V} \rightarrow \mathbb{R}$. Регулярность отображения $V$ равносильна тождеству

$$
x^{2} y=s(x) x y,
$$


которое по определению означает, что алгебра $A_{V}$ регулярна. Легко видеть, что из тождества (2) вытекает (1), однако обратное неверно, начиная с $n=3$.

Имеется одно существенное условие, которое влечет за собой адекватную генетическую интерпретацию, по крайней мере в пределах регулярности.

Стохастическое квадратичное отображение $V$ и соответствующая алгебра $A_{V}$ называются нормальнылми, если для всех $j, l$ (при $j \neq l$ ) квадратичные формы $x_{j}^{\prime}, x_{l}^{\prime}$ линейно независимы и нет таких пар $i, k$ (при $i \neq k$ ), что все $x_{j}^{\prime}$ зависят только от $x_{i}+x_{k}, x_{l}(l \neq i, l \neq k)$. Поскольку это свойство необходимо для всех генетически оправданных ситуаций (обсуждение этого обстоятельства см. в [8]) мы должны распространить его на все $V$-инвариантные грани исходного симплекса. Если это выполнено, то отображение $V$ называется ультранормальныл. В терминах алгебры $A_{V}$ ультранормальность равносильна нормальности всех координатных подалгебр.

Подчеркнем, что нормальность - это своего рода неприводимость. В самом деле, если отображение $V$ не является нормальным, то имеется естественный способ редуцировать его к нормальному отображению $\Delta^{\nu-1} \rightarrow \Delta^{\nu-1}, \nu<n, \mathrm{c}$ сохранением всех существенных основных объектов (неподвижные точки, инвариантные линейные формы и т.д.), см. [8, §3.9].

Неподвижные точки отображения $V$ - это в точности неотрицательные ненулевые идемпотенты алгебры $A_{V}$. Для каждой бернштейновской алгебры $A$ многообразие $\operatorname{Id}_{1} A$ ненулевых идемпотентов совпадает с графиком (неоднородного) квадратичного отображения $U \rightarrow A$, где $U-$ некоторое подпространство, $\operatorname{dim} U=\operatorname{dim}\left(\operatorname{Id}_{1} A\right)=m-1$. (Фактически в качестве $U$ можно взять любое подпространство, касательное к $\operatorname{Id}_{1} A$ в какой-нибудь точке.) Пара чисел $(m, \delta)$, где $\delta=n-m$, называется mипом алгебры $A$. Пространство $J$ всех инвариантных линейных форм не более чем $m$-мерно, причем $\operatorname{dim} J=m$ тогда и только тогда, когда алгебра $A$ регулярна. Если алгебра $A$ ядерна, т.е. $A^{2}=A$, то для регулярности достаточно выполнения неравенства

$$
\delta-(m-1)(m-2) / 2>0 .
$$

Кроме того, все ядерные бернштейновские алгебры с $m \leqslant 2$ или $\delta \leqslant 1$ (в частности, при $n \leqslant 4)$ регулярны, см. [8, §3.4].

В 1977 г. мы высказали предположение, что все ультранормальные стохастические бернштейновские отображения (алгебры) регулярны. Эта гипотеза была доказана нами при условии, что $m \leqslant 3$ или $\delta \leqslant 1$ (в частности, при $n \leqslant 5)$, см. [7] и [8, §5.7]. Доказательство было основано на одной комбинаторнотопологической конструкции, которая приводит к следующему предложению.

ПРЕДЛОЖЕНИЕ. В каноническом базисе $\left\{e_{1}, \ldots, e_{n}\right\}$ каждой ультранормальной стохастической бернштейновской алгебры имеется не более идемпотентов.

Теперь мы в состоянии усовершенствовать этот подход для доказательства гипотезы в общем случае.

ТЕоремА. Каждая ультранормальная стохастическая бернштейновская алгебра А регулярна.

Доказательство проводится индукцией по $n=\operatorname{dim} A$ и основано на следуюших леммах. 
ЛЕмма 1. Наименьшая координатнал подалгебра, содержащая все базиснье идемпотенты, совпадает $с$ A.

Пусть $\left\{e_{1}, \ldots, e_{\rho}\right\}$ - эти идемпотенты, и пусть $\nu_{i j}$ - размерность наименьшей координатной подалгебры, содержащей $e_{i}$ и $e_{j}(1 \leqslant i<j \leqslant \rho)$. Ясно, что $\nu_{i j} \geqslant 2$.

ЛЕмма 2. Каждое $\nu_{i j}$ меньше или равно 4.

Лемма 3. Если $\rho=n$, то все $\nu_{i j}$ равны 4 (за исключением тривиального случал, когда $\left.x^{2}=s(x) x\right)$. Если $\rho<n$, то $\nu_{i j} \leqslant 3$.

Первый случай является «дискретным» в том смысле, что множество таких алгебр конечно при каждом $n$. Второй случай, напротив, является «континуальным». На самом деле это как раз случаи әлементарной и неэлементарной генной структуры, единственно возможные в регулярной ситуации [8, гл. 4].

Комбинируя полученный результат с явным описанием всех нормальных стохастических регулярных алгебр [8, теоремы 4.3.2 и 4.6.1], мы приходим к решению проблемы Бернштейна в ультранормальном случае.

\title{
ЛитературА
}

1. Бернштейн С. Н. Наука на Украине, вып. 1, 1922, с. 14-19. 2. Bernstein S. N. C. R. Acad. Sci. Paris, 177, 528-531 (1923). 3. Bernstein S. N. C. R. Acad. Sci. Paris, 177, 581-584 (1923). 4. Бернштейн C. Н. Уч. зап. н.-и. каф. Украины, 1, 83-115 (1924). 5. Hardy G. H. Science, 28, No. 706, 49-50 (1908). 6. Любич Ю. И. УМН, 26, вып. 5, 51-116 (1971). 7. Любич Ю. И. УМН, 34, вып. 6, 53-58 (1979). 8. Любич Ю. И. Математические структуры в популяционной генетике. Наукова думка, Киев, 1983. 9. Weinberg W. Jahresber. Ver. Vaterl. Naturk. in Würtemb., 64, 368-372 (1908).

Department of Mathematics

Technion, Haifa, Israel

Поступило в редакцию 15 июня 1996 г.

УДК 517.94

\section{$L^{p}$-свойства решений квазидифференциальных уравнений и возмущение их коэффициентов на множествах положительной меры ${ }^{\star}$}

\author{
(c) 1997. К. А. МиРзоев
}

Пусть вещественные функции $p_{0}, \ldots, p_{n-1}, 1 / p_{n}(n \geqslant 1)$ измеримы на полуоси $[0,+\infty)$ и суммируемы на каждом ее подынтервале $[\alpha, \beta]$. Как обычно, символом $L^{p}(0, \infty)(1 \leqslant p \leqslant+\infty)$ обозначим пространство всех классов попарно почти всюду равных между собой измеримых функций $f$, для которых $|f|^{p}$ интегрируема на $[0,+\infty)$, если $1 \leqslant p<+\infty$, и $f$ существенно ограничена на $[0,+\infty)$, если $p=+\infty$, а символом $l_{n}[f]$ - линейное симметрическое квази-

* Настоящее исследование поддержано грантами N3I000 и N3I300 Международного научного фонда и Российского правительства. 